\title{
Numerical simulation of continuum generation in a multimode nonlinear waveguide
}

\author{
T. Chaipiboonwong, P. Horak, J. D. Mills, and W.S. Brocklesby \\ Optoelectronics Research Centre, University of Southampton, Southampton SO17 1BJ, United Kingdom
}

Continuum generation in large multimode nonlinear waveguides offers the exciting prospect of a new generation of compact, integrated, and high-power broadband light sources. Recently a novel technique based on Scanning Near-field Optical Microscopy (SNOM) has been adopted for the observation of continuum generation in such systems with subwavelength resolution. ${ }^{1}$ This revealed several interesting aspects of continuum generation, e.g., that the spectral broadening induced by optical nonlinearity depends not only on the distance of propagation but also on the location across the waveguide. Moreover the spectral broadening along the waveguide is slower than in the case of a singlemode waveguide and the spectral width may even decrease over short distances. We have now developed a multimode numerical simulation for nonlinear pulse propagation in order to explain such features.

Our simulations are based on the pulse propagation equation adapted for an N-mode field,

$$
\frac{\partial A_{m}(z, t)}{\partial z}+\beta_{1, m} \frac{\partial A_{m}(z, t)}{\partial t}+\frac{i \beta_{2, m}}{2} \frac{\partial^{2} A_{m}(z, t)}{\partial t^{2}}=i\left[\sum_{n=1}^{N} \gamma_{m n}\left|A_{n}(z, t)\right|^{2}\right] A_{m}(z, t),
$$

where $A_{m}(z, t), \quad \beta_{1, m}$ and $\beta_{2, m}$ are the slowly varying field envelope, group velocity parameter, and GVD parameter for mode $m$, respectively. The coupling parameters $\gamma_{m n}$ depend on the nonlinear refractive index $n_{2}$ of the guide material and on overlap integrals of the transverse mode intensities. Note that in Eq. (1) we only retain self- and cross-phase modulation terms which proved to be the most important nonlinear effects in our system.

The waveguide in the simulation is a rectangular $\mathrm{Ta}_{2} \mathrm{O}_{5}$ film layer $0.5 \mu \mathrm{m}$ high and $4.2 \mu \mathrm{m}$ wide on a $\mathrm{SiO}_{2}$ substrate. The dispersion constants and transverse field distributions are determined by the effective index method ${ }^{2}$ and the pulse propagation simulation is performed using the split-step Fourier method. The structure supports around twenty modes at the pump wavelength of $800 \mathrm{~nm}$. Figure 1 shows the spectral evolution along the propagation distance of 6 $\mathrm{mm}$ if the input pulse is coupled into one mode (TM00), three modes (TM00, TM10, TM20 with relative intensities 0.8 , $0.1,0.1$ ), and five modes (TM00, TM10, TM20, TM30, TM40 with relative intensities $0.7,0.1,0.1,0.05,0.05$ ), respectively. The simulation calculates the field collected by a SNOM tip of $100 \mathrm{~nm}$ diameter placed $20 \mathrm{~nm}$ above the centre of the guide and moving along the length of the guide. The spectra exhibit clear multimode interference, as well as slower spectral broadening in the multimode case caused by the temporal walk-off of higher-order modes. The three mode-mixing spectral variation across the $4 \mu \mathrm{m}$ width of the waveguide after $5 \mathrm{~mm}$ propagation distance is shown in Figure 2. The rich transverse spatial structure of higher-order modes leads to noticeable fluctuations of the spectral width and of the peak wavelength from one side of the guide to the other.

In conclusion, we have developed a numerical model which explains some of the most striking phenomena in continuum generation in multimode nonlinear optical waveguides as observed by SNOM.

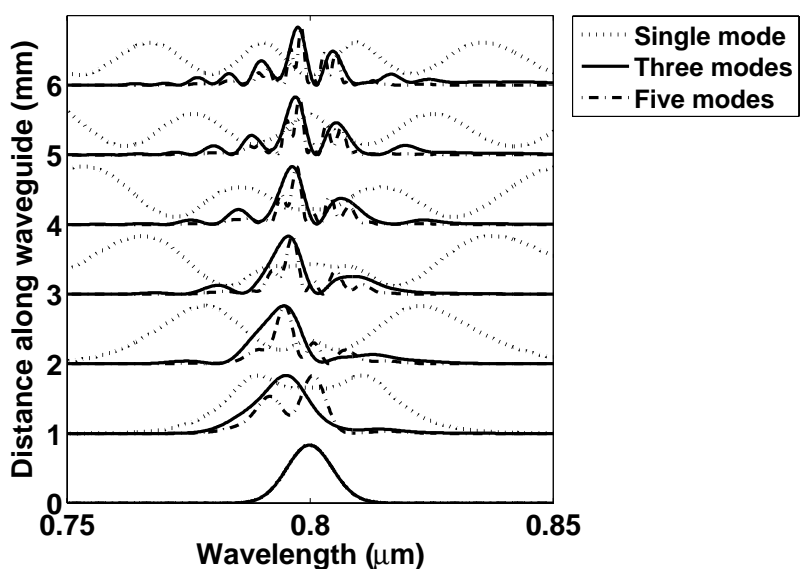

Figure 1 Spectral evolution along the propagation distance

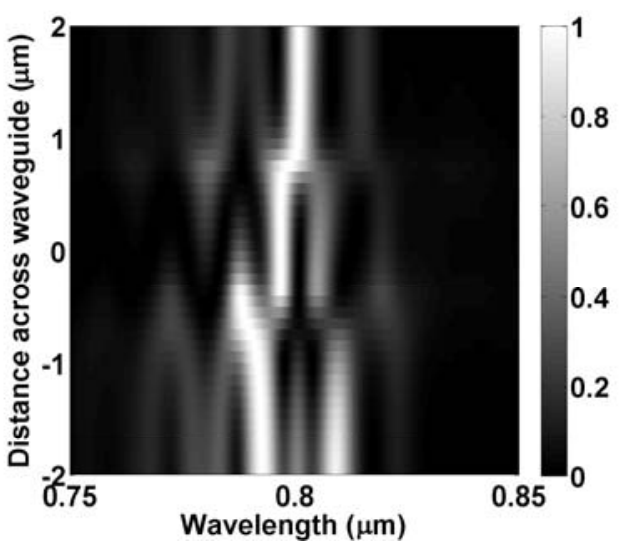

Figure 2 Spectral variation across the waveguide

\section{References}

1. J. Mills et al., "Observation of the developing optical continuum along a nonlinear waveguide," Opt. Lett. 31, 24592461 (2006).

2. K. Chiang et al., "Effective-index method with built-in perturbation correction for integrated optical waveguides," J.

Lightwave Technol. 14, 223-228 (1996). 